\title{
A numerical algorithm for run-curve optimization of trains considering a DC feeding circuit
}

\author{
H. Ko \& M. Miyatake \\ Department of Electrical and Electronics Engineering, \\ Sophia University, Japan
}

\begin{abstract}
An algorithm optimizing total energy consumption of multiple train operation considering a DC feeding circuit is investigated in this paper. Our mathematical formulation includes several characteristics of trains which depend on feeding voltage. It makes it possible to give detailed consideration to an energy-saving operation. It is especially important for us to be able to discuss the influence of squeezing control of regenerating current and feeding loss. We constructed the optimizing algorithm based on the gradient method applicable to large-scale problems for future works. Several numerical examples are demonstrated to verify the reliability and validity of the proposed method. Every optimisation result is obtained within a minute.
\end{abstract}

Keywords: train operation control, optimization, energy-saving, DC feeding system.

\section{Introduction}

In recent years, regenerative energy of trains with electrical regenerative braking system plays an important role in reducing whole energy consumption in DC railway system. However, there are several difficulties in collecting regenerative energy efficiently. One of these difficulties is that the regenerating ability and amount of regenerating energy depend closely on feeding voltage of a train. For example, the maximum amount of the electrical braking torque is limited by feeding voltage, and feeding loss also relies on feeding voltage. In addition, it is indispensable to discuss influence of squeezing control of regenerative current for preventing overvoltage of feeder. The other difficulty in collecting 
regenerative energy is preparing external load for absorbing regenerating energy, e.g. the other accelerating trains. This consideration conducts discussion for energy-saving operation towards an idea that acceleration/deceleration timings of several trains are important to reuse regenerative energy efficiently, in other words, optimizing several trains operation is required.

We already realized to solve the kinetic energy minimization problem of a train in previous works [1-3]. However, we did not consider the effect of energy exchange among multiple trains, because of difficulties in mathematical formulation and developing numerical algorithm.

In this paper, we formulate the energy-saving operating problem of several trains considering DC feeding system as an optimal control problem. The proposed formulation includes equality constraints of circuit equations of a feeding system. We also propose a simplified parallel numerical optimizing algorithm based on the traditional gradient method step by step. We verify the reliability and validity of the proposed method by some numerical examples about two trains operation.

\section{Mathematical formulation}

In this section, the energy-saving operation problem of trains is described as an optimal control problem. The variable vector $\zeta(t)$ is used for whole state vectors of system (position and speed of trains, node voltages of a feeding circuit, control inputs, and so on) in this paper.

\subsection{Objective functional}

The objective functional $J$ is given as the total electrical energy consumption at substations in eqn (1).

$$
J=\int_{0}^{T} \sum_{m=1}^{M} E_{m}(t) I_{m}(\zeta(t), t) d t
$$

Here, $T$ is total time, $M$ is the number of substations. Substation source voltage $E_{m}$ and substation load current $I_{m}$ are defined as time-dependent function, load current $I_{m}$ relies on system variables $\zeta$.

In previous works, objective functional was chosen as total kinetic energy consumption, under a given feeding voltage variation $[2,3]$. On the contrary, the objective functional $J$ is preferable to be formulated as eqn (1) in our case, because we can consider feeding loss and effect of squeezing control with given functional eqn (1).

\subsection{Trains}

We formulate states and restrictions on train operation as state equations and inequality constraints in an optimal control problem, respectively. State 
equations and constraints of $j$-th train are given as eqn (2) using position $x_{j}$, speed $v_{j}$, feeding voltage $e_{j}$, departure time $t_{j 0}$, arrival time $t_{j f}$, departure position $x_{j 0}$, arrival position $x_{j f}$, speed limit $v_{\text {jmax }}$, maximum acceleration with electrical torque $f_{\text {jmax }}$, maximum deceleration with electrical and mechanical blending torque $f_{\text {jmin }}$, and load current $i_{j}$ of a train.

$$
\begin{gathered}
\dot{x}_{j}=\left\{\begin{array}{cc}
v_{j} & t_{j 0} \leq t \leq t_{j f} \\
0 & \text { others }
\end{array}\right. \\
\dot{v}_{j}=\left\{\begin{array}{c}
f_{j}\left(u_{j}, e_{j}, v_{j}\right)-r_{j}\left(x_{j}, v_{j}\right) \quad t_{j 0} \leq t \leq t_{j f} \\
0 \\
\text { others }
\end{array}\right. \\
x\left(t_{j 0}\right)=x_{j 0}, \quad v\left(t_{j 0}\right)=0 \\
x\left(t_{j f}\right)=x_{j f}, \quad v\left(t_{j f}\right)=0 \\
f_{j \min } \leq f_{j}\left(u_{j}, e_{j}, v_{j}\right) \leq f_{j \max }\left(e_{j}, v_{j}\right) \\
v_{j} \leq v_{j \max }\left(x_{j}\right) \\
i_{j}=i_{j}\left(u_{j}, e_{j}, v_{j}\right)
\end{gathered}
$$

Eqn (2) and (3) are kinetic equations, eqn (4) and (5) are boundary conditions at departure/arrival time. Here, function $f_{j}$ describes acceleration/deceleration and $u_{j}$ is control input defined as table 1. Torque and speed limit of a train is described as eqn (6) and (7). Maximum acceleration $f_{\text {jmax }}$ varies according to train speed and catenary voltage (please see figure 2(a)). Function $r_{j}$ consists of running and grade resistance. Eqn (8) means load current of a train depends on kinetic power and feeding voltage. It implies that we can consider influence of squeezing control and limitation of regenerative electrical torque, too. We also assume that the mechanical braking torque is only used when the required braking torque exceeds the maximum electrical braking torque for energy-saving operation.

Table 1: $\quad$ Definition of control input $u$.

\begin{tabular}{|l|l|}
\hline$u=-1$ & maximum deceleration \\
\hline$u=-1$ to 0 & deceleration, the amount of brake torque is proportional to $-u$ \\
\hline$u=0$ & coasting \\
\hline$u=0$ to 1 & acceleration, the amount of motive torque is proportional to $u$ \\
\hline$u=1$ & maximum acceleration \\
\hline
\end{tabular}

\subsection{Feeding circuit}

We regard circuit equations of a feeding system as equality constraints in the optimal control problem shown as eqn (9) except for transient responses in and under millisecond region. 


$$
C_{l}(\zeta(t), t)=0 \quad(l=1, \cdots, P)
$$

Here, $P$ is the number of circuit equations. In general, circuit equations of a feeding system are too complicated to solve analytically. Thus, a numerical optimization algorithm which does not require analytically solvable equality constraints is suitable for the optimal control problem of trains considering feeding system.

\subsection{Representation as optimal control problem}

Finally, we formulate the energy-saving operating problem of trains as an optimal control problem (10) with equality and inequality constraints considering previous sections 2.1 2.3. The variable $N$ indicates the number of trains.

$$
\min _{\left\{u_{j}\right\}_{j=1, \cdots, N}} J, \quad \text { subj.to }(2), \cdots,(9)
$$

We assume every function in eqn (10) is differentiable using the mollifier if needed. This optimal control problem (10) could be a large-scale problem.

\section{Numerical algorithm}

In this section, a numerical algorithm for the formulated problem (10) is derived using some transformation techniques and the optimality condition. The variables without lower position indexes mean sets of the all elements corresponding variables (for example, $u=\left\{u_{1}, u_{2}, \ldots\right\}$ ).

\subsection{Optimality and fundamental algorithm}

The formulated optimal control problem (10) is so called a two-point boundary value problem which has constraints on initial state variables (4) and terminal states (5). It is generally considered that an optimal control problem of this class meets any difficulties to solve numerically. A transformation technique to initialvalue problem is employed to avoid these difficulties. The modified objective function is derived as eqn (11).

$$
J^{\prime}=J+\sum_{j=1}^{N}\left\{p_{j 1}\left(x\left(t_{j f}\right)-x_{j f}\right)^{2}+p_{j 2} v\left(t_{j f}\right)^{2}\right\}
$$

Here, $p_{j 1}$ and $p_{j 2}$ are designed penalty parameters adjusted by trial and error task, but it is easy to perform an automated adjustment algorithm [6].

The other transformation from constrained input problem to unconstrained one employing slack control input $u^{\prime}$ ( $\operatorname{such}$ as $\left.u=\sin \left(u^{\prime}\right)\right)$ is also used to simplify the formulated optimal control problem (10). Finally, the simplified optimal 
operating problem (12) is derived from these transformation techniques and assumption that the solution of circuit equation (9) is unique.

$$
\begin{array}{ll}
\min _{\left\{u_{j}^{\prime}\right\}_{j},\left\{e_{l}\right\}_{l}} J^{\prime} & \\
\text { subj.to } & \dot{\xi}_{j}=g_{j}\left(u_{j}^{\prime}, \xi_{j}, t\right), \quad \xi_{j}\left(t_{j 0}\right)=\xi_{j 0} \quad(j=1, \cdots, N) \\
& C_{l}(\zeta, t)=0 \quad(l=1, \cdots P)
\end{array}
$$

Here, the state vector $\xi_{j}=\left(x_{j}, v_{j}\right)^{T}$ and the vector function $g_{j}$ are introduced to simplify the expression. The vector function $g_{j}$ consists of the right hand side of eqn (2) and (3). The variable $e_{l}$ is assumed as quasi-control-input to solve the circuit equations (9) numerically.

The optimality condition of problem (12) is given as follows [4].

$$
\begin{gathered}
H_{u_{j}^{\prime}} \equiv \partial H / \partial u_{j}^{\prime}=0 \\
H_{e_{l}} \equiv \partial H / \partial e_{l}=0
\end{gathered}
$$

The Hamiltonian $H$ is defined as eqn (15) and (16) introducing variable vector $\lambda_{j}, \pi_{l}$.

$$
\begin{gathered}
H=\sum_{m=1}^{M} E_{m} I_{m}+\sum_{j=1}^{N} \lambda_{j}{ }^{T} g_{j}+\sum_{l=1}^{P} \pi_{l} C_{l} \\
\dot{\lambda}_{j}=-\partial H / \partial \xi_{j}
\end{gathered}
$$

It is a well-known result as one of the optimal control theories that the function $H_{u}$, presents gradient function which gives the steepest ascending direction. Thus, the numerical optimization algorithm for problem (12) is given as follows considering that the vector $\pi_{l}$ is found as solution of equality (14).

$<$ Algorithm I $>$

(a) give the initial estimation $u^{0}, e^{0}$, and set $k=1$.

(b) solve ODE(Ordinal Differential Equation)s (2),(3) and (4) using $u^{k}, e^{k}$, and obtain $\zeta^{k}$.

(c) solve ODE (16) and equalities (14), and obtain $\lambda^{k}, \pi^{k}$.

(d) calculate gradient function by (13), using $\zeta^{k}, \lambda^{k}, \pi^{k}$.

(e) execute line search toward the search direction (steepest gradient direction, conjugate gradient direction, and so on), and set the solution $u^{k+1}$.

(f) solve equality (9), and set the solution as $e^{k+1}$

(g) if the difference between $e^{k}$ and $e^{k+1}$ is adequately small, the procedure terminates, or else set the $k=k+1$ and return to step (b). 
This algorithm is based on the traditional gradient method for unconstrained optimal control problem.

\subsection{Parallel algorithm}

As mentioned in section 2.4, the formulated optimal train operating problem (10) (or eqn (12)) could be a large-scale problem. Parallel algorithms are valuable because these are faster to perform large computing task. A parallel algorithm for the formulated problem is prepared employing Kirchhoff's Current Law (KCL) as follows. The objective functional $J$ is expanded with KCL as eqn (17).

$$
J=\int_{0}^{T}\left(\sum_{j=1}^{N} \alpha_{j} i_{j}+\sum_{s} \alpha_{s} i_{s}\right) d t
$$

Here, $\alpha_{j}$ and $\alpha_{s}$ are the coefficients derived by expansion, and the variable $i_{s}$ is a current without load currents of trains. Thus, the objective functional $J$ is divided for every train shown as eqn (18) distributing the terms which do not involve load current properly.

$$
\begin{aligned}
J & =\sum_{j=1}^{N} \int_{0}^{T}\left(\alpha_{j} i_{j}+\sum_{q} \sigma_{q}\right) d t \\
& \equiv \sum_{j=1}^{N} U_{j}
\end{aligned}
$$

Here, variable $\sigma_{q}$ is the term belonging to $U_{j}$.

Then, a parallel algorithm for the formulated problem (12) is performed as the following sequence.

$<$ Algorithm II $>$

(a) give the initial estimation $u^{0}, e^{0}$, and set $k=1$.

(b) solve ODEs (2),(3) and (4) using $u^{k}, e^{k}$, and obtain $\zeta^{k}$.

(c) solve ODE (16) and equalities (14), and obtain $\lambda^{k}, \pi^{k}$.

(d) calculate gradient function for every train, using $\zeta^{k}, \lambda^{k}, \pi^{k}$.

(e) execute line search in the search direction for every train, and set the solution $u^{k+1}$.

(f) solve equality (9), and set the solution as $e^{k+1}$.

(g) if the difference between $e^{k}$ and $e^{k+1}$ is adequately small, the procedure terminates, or else set the $k=k+1$ and return to step (b).

In this algorithm, step (d) and (e) could be proceeded simultaneously for every train. Namely, the original optimal control problem is distributed for every train and interactions between trains are mainly informed by $e, \pi$. 


\subsection{Simplified algorithm}

The parallel algorithm shown in subsection 3.2 could be valuable for a largescale problem formulated as eqn (10). However, this algorithm would still have some difficulties. The variable vector $\pi$ is found as solution of eqn (14) mentioned above. But, the equality (14) can become numerically unstable or its solutions are not unique because of the complexity of the feeding circuit such as characteristics of diode rectifier. For this reason, we propose the following intuitive approximated method.

$<$ Algorithm III $>$

(a) give the initial estimation $u^{0}, e^{0}$, and set $k=1$.

(b) solve ODEs (2),(3) and (4) using $u^{k}, e^{k}$, and obtain $\zeta^{k}$.

(c) solve ODE (16) neglecting the terms including $\pi^{k}$, and obtain $\lambda^{k}$.

(d) calculate gradient function for every train neglecting the terms including $\pi^{k}$, using $\zeta^{k}, \lambda^{k}$.

(e) execute line search in the search direction for every train, and set the solution $u^{k+1}$.

(f) solve equality (9), and set the solution as $e^{k+1}$.

(g) if the difference between $e^{k}$ and $e^{k+1}$ is adequately small, the procedure terminates, or else set the $k=k+1$ and return to step (b).

This simplified algorithm does not satisfy the optimality condition of the original problem (10), but step (b), (c), (d) and (e) could be proceeded simultaneously for every train, and the solution of equality (14) is not required. Namely, this algorithm is more suitable for large-scale problems and numerically stable than Algorithm II. Algorithm III is performed based on the assumption that interactions between trains are mainly informed with feeding voltage $e$.

\section{Numerical examples}

In this section, three numerical examples of two-train-operation in DC feeding system shown as figure 1 are demonstrated using Algorithm III. Please refer [6] for comparison between Algorithm II and III. In the following examples, conjugate gradient direction is employed as a search direction in line search, and Clipping-Off algorithm [5] is used to deal with inequality constraints on control inputs instead of slack control inputs. It does not cause any essential difference from our proposed method. The $\mathrm{C}++$ language on an Intel Celeron $1.4 \mathrm{GHz}$ machine is employed for demonstrations.

The characteristics of motive and brake torque, and squeezing control are presented in figure 2 . The supply voltage of substations $E_{s}$, internal resistance of substations $R_{s}$ and line resistance $R_{l}$ is given as $1500[\mathrm{~V}], 0.05[\Omega]$ and $0.04[\mathrm{~m} \Omega / \mathrm{m}]$, respectively. All trains transfer $2180[\mathrm{~m}]$ in $130[\mathrm{sec}]$ between stations. The first train departs from the position $x=0[\mathrm{~m}]$ at time $t=0[\mathrm{sec}]$, and the second train departs from the position $x=2180[\mathrm{~m}]$ at time $t=t_{s}=90,65$ or $40[\mathrm{sec}]$. The departing time of the second train is changed in order to change the phase of two trains. 


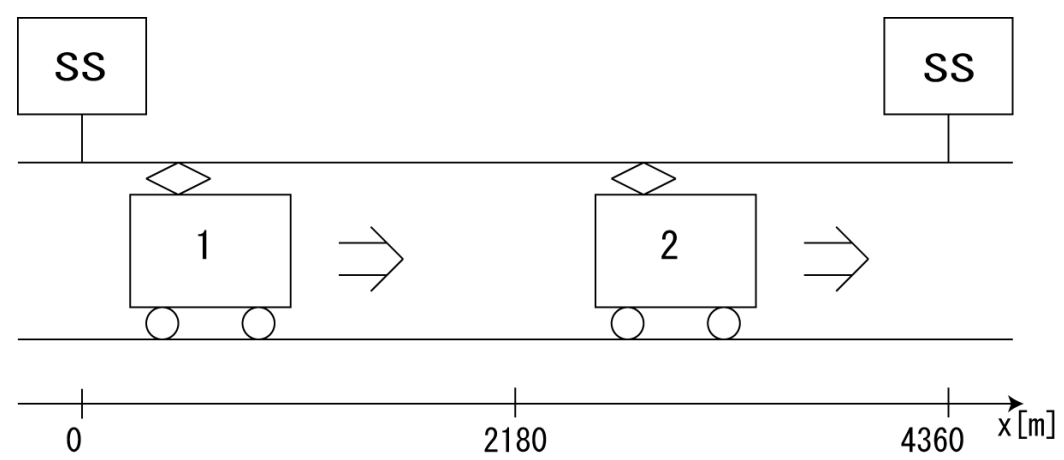

Figure 1: Schematic model of numerical examples.

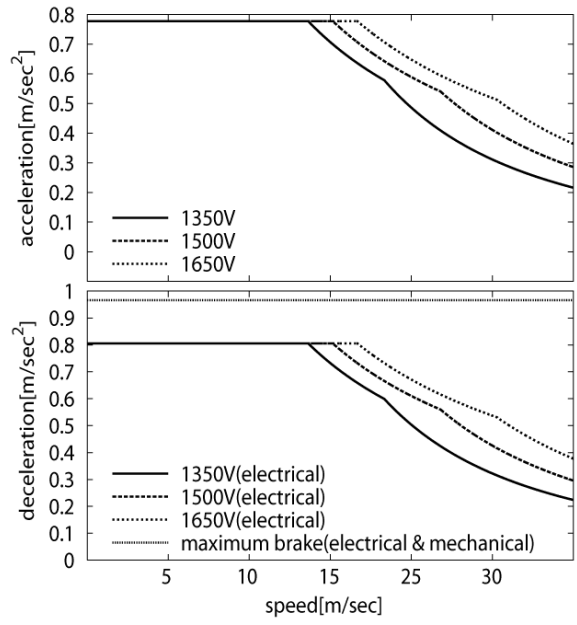

(a) acceleration/deceleration

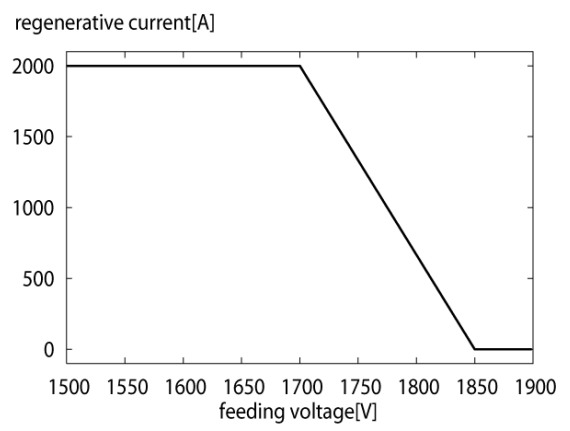

(b) squeezing control

Figure 2: Characteristics of a train.

The optimization results of these three cases are depicted in figure 3, in which horizontal axis is chosen as the normalized time $\tau=t / T$. The optimal energy consumptions are found as (a) $244.1[\mathrm{MJ}]\left(t_{s}=90\right)$, (b) $280.4[\mathrm{MJ}]\left(t_{s}=65\right)$, (c) $329.1[\mathrm{MJ}]\left(t_{s}=40\right)$. Each optimizing procedure is converged in less than 1 minute.

All the results indicate that the first train acts like an energy storage device. The first train can collect electrical energy with comparatively high efficient near the departure position, on the contrary, greater feeding loss occurs when the second train starts. Thus, the first train accelerates up to higher speed to store electrical energy as kinetic energy in advance, and supplies the stored energy when the second train accelerates in order to reduce feeding loss. The amount of braking torque of the first train is adjusted to minimize the effect of squeezing control, if the maximum braking torque were employed in higher speed area, the amount of regenerative energy is cut down as the following squeezing 
characteristics shown in figure 2 (b). Comparison task among the optimization results with three different departure time of the second train shows when the proper time is to use regenerative energy most efficiently for energy-saving operation. If the departure time of the second train is preferable $\left(t_{s}=90\right)$, braking interval of the first train and accelerating interval of the second train is the same. In other cases $\left(t_{s}=65\right.$ or 40$)$, regenerative energy of the first train is used for accelerating stage of the second train in comparatively high-speed area because electrical power demand and feeding loss become greater at this point.
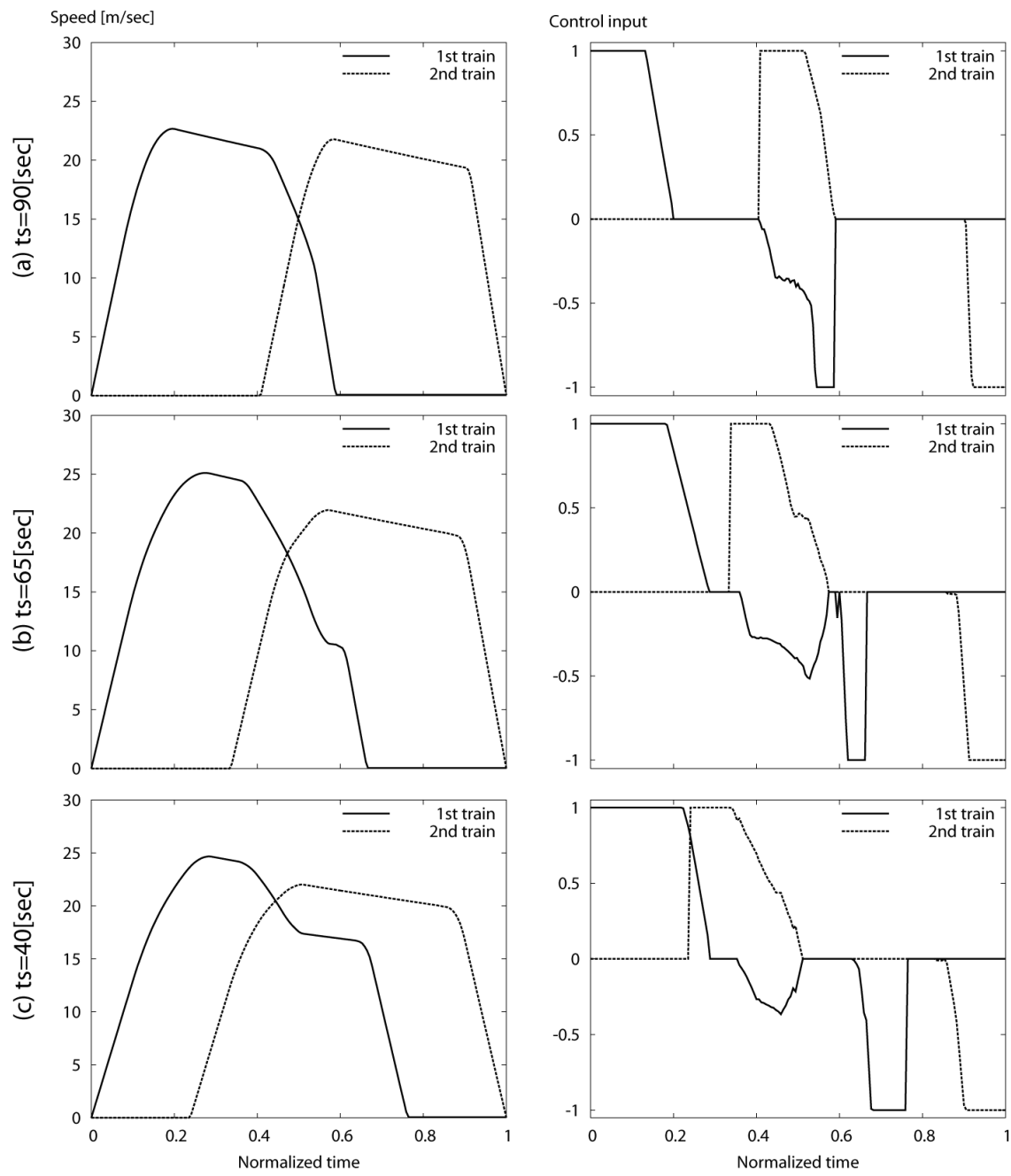

Figure 3: Optimization results with different departing phase.

These considerations for energy-saving operation were already discussed in our previous works. However, we only mentioned qualitative analysis, not based 
on the quantitative optimal solutions. Our newly proposed formulation and numerical method could overcome these insufficient discussions and make important role at this point.

\section{Conclusion}

The optimal operating problem of multiple trains considering DC feeding system is formulated and the simplified approximated numerical algorithm is performed. We can find that the proposed method has enough reliability and performance from numerical examples of two trains. We also show that the influence of operating phase between two trains can be evaluated quantitatively. Further validation using models with more than two trains and energy storage devices would be our future works.

\section{References}

[1] Khmelnitsky, E., An Optimal Control Problem of Train Operation. IEEE Trans. on Automatic Control, vol.45, No.7, pp.1257-1266, 2000.

[2] Ko, H., Miyatake, M. \& Koseki, T., Application of Dynamic Programming to Optimization of Running Profile of A Train, Comprail2004, pp.103-112.

[3] Ko, H. \& Miyatake, M., Fundamental Study on Train Run-curve Optimization Problem Employing Gradient Method, National Convention of IEEJ, vol.4, pp378-379, 2005 (in Japanese).

[4] Kanou, H., Theory and Computational Methods in Optimization, 1987 (in Japanese).

[5] Quintana, V.H. \& Davison, E.J., Clipping-off Gradient Algorithm to Compute Optimal Controls with Constrained Magnitude, Int. J. Control, vol.20, No.2, pp243-255.

[6] Ko, H. \& Miyatake, M., A Numerical Method for Run-curve Optimization of Trains Considering Feeding Circuit, TER05-35, pp31-36, 2005 (in Japanese). 